\title{
Hepatic perivascular epithelioid cell neoplasm: A clinical and pathological experience in diagnosis and treatment
}

\author{
WENYING CHEN $^{1 *}$, YEQING LIU ${ }^{2 *}$, YANYAN ZHUANG ${ }^{1}$, JUANFEI PENG ${ }^{1}$, \\ FENGTING HUANG $^{1}$ and SHINENG ZHANG ${ }^{1}$
}

\begin{abstract}
Departments of ${ }^{1}$ Gastroenterology and Guangdong Provincial Key Laboratory of Malignant
Tumor Epigenetics and Gene Regulation, and ${ }^{2}$ Pathology, Sun Yat-sen Memorial Hospital, Sun Yat-sen University, Guangzhou, Guangdong 510120, P.R. China
\end{abstract}

Received October 17, 2016; Accepted December 19, 2016

DOI: $10.3892 / \mathrm{mco} .2017 .1168$

\begin{abstract}
Hepatic perivascular epithelioid cell neoplasm (PEComa) is a rare type of neoplasm derived from mesenchymal tumors that is often misdiagnosed as hepatocellular carcinoma (HCC), hepatic hemangioma or other liver malignancies. The clinical and histological characteristics of PEComa have yet to be fully documented. To optimize the diagnosis and treatment of the disease, a retrospective analysis was performed to investigate the clinicopathological characteristics of 7 patients diagnosed with hepatic PEComa in the Sun Yat-Sen Memorial Hospital between January 2004 and December 2015. Briefly, all the patients lacked specific symptoms, and a serological examination provided no further useful information. Additionally, non-specific imaging manifestations were observed. Microscopically, detection of epithelioid or spindle-shaped cells with adipocytes was suggestive of the disease, and an analysis of biomarkers, including the monoclonal antibody HMB-45, the melanocytic differentiation marker, Melan-A, and smooth muscle $\alpha$-actin (SMA), helped to confirm the diagnosis. Regarding the treatment, 6 patients $(85.7 \%)$ received surgical resection procedures, and 1 patient (14.3\%) was admitted for tumor arterial embolization and percutaneous microwave coagulation therapy. After a follow-up period of 12-20 months, no recurrence was observed. Taken together, hepatic PEComa should be suspended in patients with a liver tumor with asymptomatic manifestation and normal
\end{abstract}

Correspondence to: Professor Shineng Zhang or Dr Fengting Huang, Department of Gastroenterology and Guangdong Provincial Key Laboratory of Malignant Tumor Epigenetics and Gene Regulation, Sun Yat-sen Memorial Hospital, Sun Yat-sen University, No. 107 Yanjiang West Road, Guangzhou, Guangdong 510120, P.R. China

E-mail: shinengz@hotmail.com

E-mail: rachelh1982@163.com

${ }^{*}$ Contributed equally

Key words: perivascular epithelioid cell neoplasm, angiomyolipoma, HMB-45, Melan-A serological test results. In imaging studies, hepatic PEComa was able to mimic HCC, hepatic hemangioma and metastatic tumor, although the mass did not invade the adjacent organs and vessel. The definitive diagnosis was made on the basis of the typical morphological features and notable markers of the tumor tissue. It is recommended that patients with PEComa in a benign pattern deserve serial imaging follow-up, but surgery is indicated in patients suffering from large tumors $(>5 \mathrm{~cm})$, or progressive enlargement or malignant tendency.

\section{Introduction}

Hepatic perivascular epithelioid cell neoplasm (PEComa) is a rare mesenchyme-derived neoplasm that is primarily composed of histologically distinctive perivascular epithelioid cells (PECs), which share the expression of the monoclonal antibody, HMB-45, smooth muscle cell markers and/or the melanocytic differentiation marker, Melan-A (1). In the majority of hepatic PEComas, adipocytes may also be found intermixing with PECs in varying proportions (2). Larger tumors may have necrosis, or be associated with hemorrhage. The imaging presentation of hepatic PEComa is highly variable, and often leads to misdiagnosis as hepatocellular carcinoma (HCC), hepatic hemangioma, or other liver tumors (3-5). The clinical and histological characteristics of PEComa have yet to be fully documented.

In the present study, the clinical data of 7 patients with hepatic PEComa admitted to the Sun Yat-Sen Memorial Hospital were retrospectively analyzed. To optimize the diagnosis and treatment of the disease, a systemic analysis was performed, highlighting the clinical, imaging and pathological characteristics.

\section{Materials and methods}

Patients and tissue specimens. Specimens from a total of 7 patients with hepatic PEComa were collected at the Sun Yat-Sen Memorial Hospital, Sun Yat-Sen University (Guangzhou, China) between January 2004 and December 2015. The samples were obtained from surgery or biopsy, with the patients' informed consent, and were histologically confirmed. All tissue samples were derived from untreated patients, fixed with formalin and embedded in paraffin. 


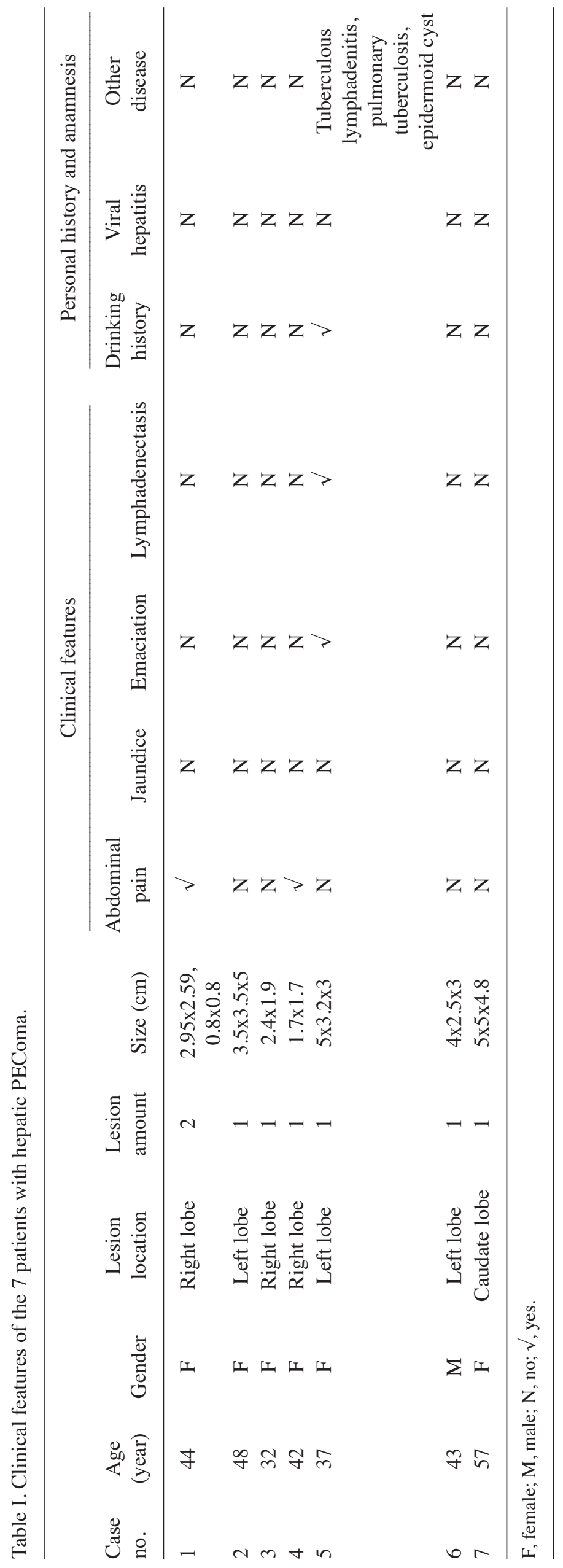


Hepatic imaging. All patients were evaluated with imaging. A total of 4 patients underwent a computed tomography (CT) scan and contrast-enhanced CT. The other 3 patients were examined with magnetic resonance imaging (MRI), and 4 patients underwent additional ultrasonography. Manifestation of the following abnormalities was recorded: Morphology of the lesion, blood vessel invasion, blood vessel displacement, bile duct invasion, cholangiectasis and lymphadenectasis.

Statistical analysis. Statistical analysis was performed using SPSS version 19.0 software (SPSS, Inc., Chicago, IL, USA). Continuous variables were compared using analysis of variance, and were expressed as the mean \pm standard deviation.

\section{Results}

Clinical data. The median age of the 7 patients with hepatic PEComa was 43 years old (range, 32-57 years), including 6 females $(85.7 \%)$ and 1 male $(14.3 \%)$. A total of 4 patients $(57.1 \%)$ were asymptomatic, and the lesions were detected incidentally during routine health check-ups. Two patients presented with abdominal discomfort (28.6\%). In the 7 th patient (14.3\%), who was suffering from turberculous lymphadenitis, the initial symptoms were emaciation and lymphadenectasis. All the patients denied having had a history of viral hepatitis or drinking, with the exception of the male patient. He had a 10-year history of alcohol abuse (Table I). Laboratory examinations revealed normal liver function, with the exception of the male with a history of alcohol abuse, who had a moderately elevated level of alanine transaminase. Levels of the tumor markers $[\alpha$-fetoprotein (AFP), carcinoembryonic antigen (CEA) and cancer antigen 19-9 (CA19-9)] were within the normal range. One patient was identified as being positive for hepatitis E virus immunoglobulin M (HEV-IgM; Table II).

A total 6 out of the 7 patients had one lesion $(85.7 \%)$; the remaining patient had two lesions (14.3\%). The tumors originated from the right liver lobe in 3 patients (42.9\%) from the left lobe in 3 patients (42.9\%), and from the caudate lobe in 1 patient (14.3\%). With respect to tumor size, 3 patients $(42.9 \%)$ had a maximum diameter of the lesion of $<3 \mathrm{~cm}$, and 4 patients $(57.1 \%)$ had tumor sizes of between 3 and $5 \mathrm{~cm}$.

Imaging characteristics. A total of 4 patients underwent CT (57.1\%); the other 3 patients were subjected to MRI (42.9\%), and for 4 of the patients (57.1\%), additional ultrasonography was performed. Generally speaking, all images demonstrated that the lesions were of an ovoid shape with well-demarcated margins, with the exception of one case subject, who exhibited no clear boundary. No blood vessel or bile duct invasion was detected; neither was any metastasis or infiltration of adjacent organs observed.

Concerning the 4 patients examined with ultrasound, 3 patients appeared with a hyperechoic mass $(75 \%)$ and 1 patient exhibited a hypoechoic mass (25\%). In the $4 \mathrm{CT}$ images, during the scanning period, low dense $(75 \%)$ or isodense $(25 \%)$ lesions were observed. Through the enhanced scanning, it was revealed that the lesion became intensely enhanced in 3 patients $(75 \%)$, with no clear enhancement observed in 1 case (25\%; Fig. 1). The images of portal and delay phase were variable. In the MRI
Table II. Demographic characteristics of the 7 patients with hepatic PEComa ${ }^{\mathrm{a}}$.

\begin{tabular}{lc} 
Characteristic & Value (mean $\pm \mathrm{S}$ \\
\hline Total no. of participants & 7 \\
Age, $\mathrm{y}$ & $43.29 \pm 7.95$ \\
Gender, female, $\%$ & $6(85.7 \%)$ \\
TBIL, $\mu \mathrm{mol} / \mathrm{l}$ & $11.40 \pm 4.47$ \\
DBIL, $\mu \mathrm{mol} / \mathrm{l}$ & $3.09 \pm 1.68$ \\
Albumin, g/l & $44.67 \pm 4.51$ \\
Hemoglobin, g/l & $128.14 \pm 4.98$ \\
ALT, U/l & $18.86 \pm 15.26$ \\
AST, U/l & $19.57 \pm 6.19$ \\
AFP, $\mu \mathrm{g} / \mathrm{l}$ & $3.06 \pm 2.03$ \\
CEA, $\mu \mathrm{g} / \mathrm{l}$ & $1.24 \pm 0.30$ \\
CA19-9, U/ml & $13.14 \pm 7.99$ \\
Viral hepatitis, positive, $\%$ & $1(14.28 \%)$
\end{tabular}

aData are presented as the mean $\pm \mathrm{SD}$ or $\mathrm{n}(\%)$. SD, standard deviation; TBIL, total bilirubin; DBIL, direct bilirubin; ALT, alanine transaminase; AST, aspartate transaminase; AFP, $\alpha$-fetoprotein; CEA, carcinoembryonic antigen; CA19-9, cancer antigen 19-9.

examination, the 3 cases all exhibited a slightly hypointense signal on the T1 weighted image (T1WI), and slight hyperintensity on the T2WI signal (100\%). However, the enhanced images were variable (Tables III and IV). Collectively, the correct diagnosis rate was only $25 \%$ within the group of patients who underwent a CT examination, and the use of ultrasonography or MRI failed to contribute to the correct diagnosis for any of the patients examined by these techniques. These cases predominantly had been mistaken as HCC or hepatic hemangioma.

Pathological features. A total of 6 patients underwent partial hepatectomy. Generally speaking, soft or firm, well-circumscribed nodular masses with a yellow, yellow-tan, gray-red or tan appearance were observed (Fig. 2). One of our cases presented a cyst inside the lesion. Microscopically, epithelioid or spindle-shaped cells were identified. Adipocytes were identified in 5 specimens (83.3\%; Fig. 3A-C). Fine needle aspiration biopsy (FNAB) was performed for 2 patients. Microscopy revealed epithelioid cells with pale, clear, eosinophilic cytoplasm or foamy cytoplasm. The nuclei were round to oval with small nucleoli, and inclusions were observed in a few of the cells (Fig. 3D). Mitotic figures were rarely identified. Finally, markedly positive staining for HMB-45, Melan-A and SMA was observed in all patients (Fig. 4).

Treatment and prognosis. A total of 6 patients received surgical treatments, including laparotomic and laparoscopic partial hepatectomy. One patient received tumor arterial embolization (TAE) and percutaneous microwave coagulation therapy (PMCT). None of the patients had received chemotherapy or radiation therapy prior to or following the tumor resection. No patient suffered from postoperative complications or died during the perioperative period. After a follow-up period of 12-20 months, no recurrence occurred. 


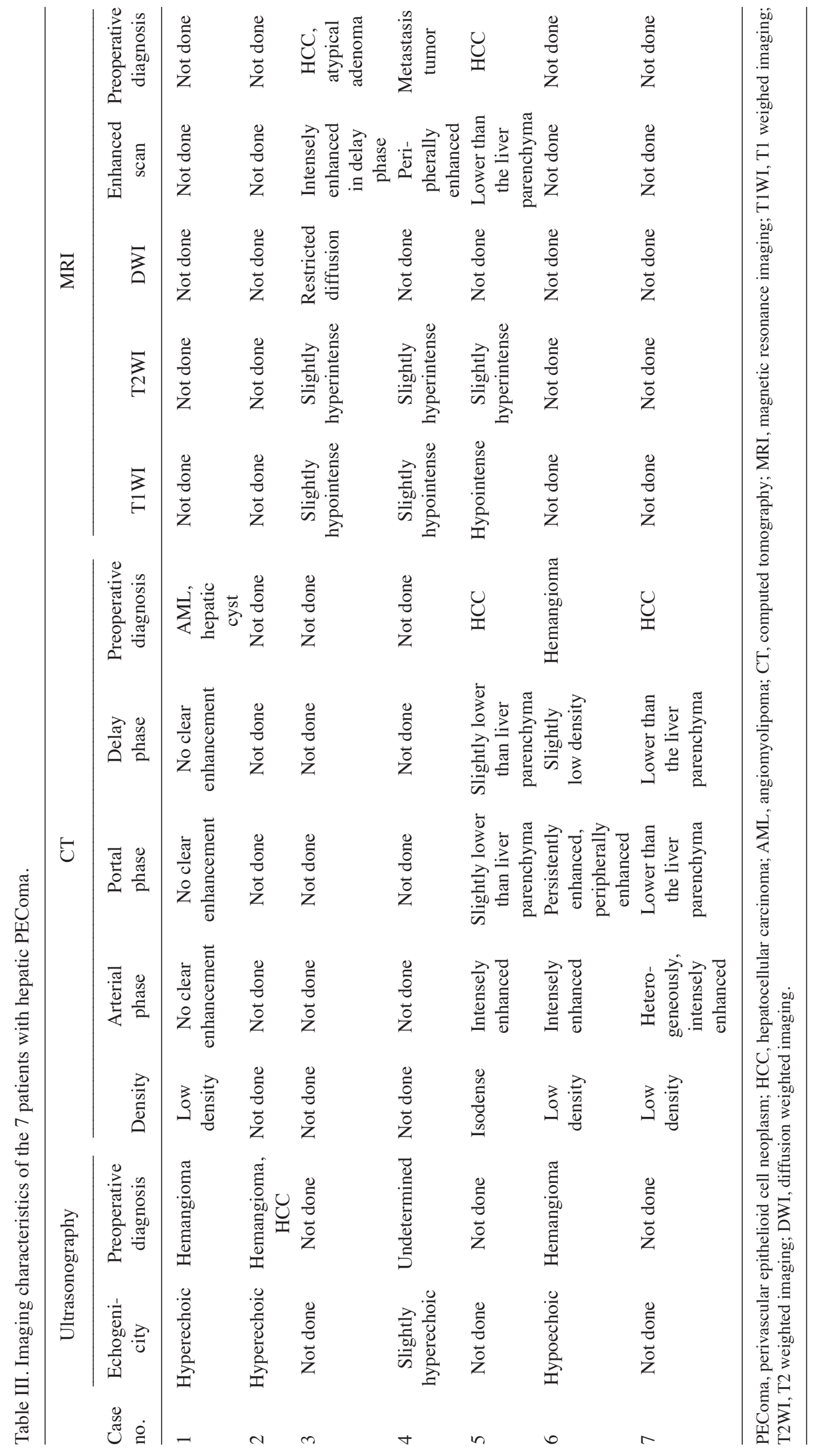


Table IV. Morphology and invasive signs of the lesions in the 7 patients with hepatic PEComa.

\begin{tabular}{|c|c|c|c|c|c|c|c|}
\hline \multirow{3}{*}{$\begin{array}{l}\text { Case } \\
\text { no. }\end{array}$} & \multirow{2}{*}{\multicolumn{2}{|c|}{ Morphology }} & \multicolumn{4}{|c|}{ Blood vessel and bile duct } & \multirow[b]{3}{*}{ Lymphadenectasis } \\
\hline & & & \multirow{2}{*}{$\begin{array}{l}\text { Blood vessel } \\
\text { invasion }\end{array}$} & \multirow{2}{*}{$\begin{array}{l}\text { Blood vessel } \\
\text { displacement }\end{array}$} & \multirow{2}{*}{$\begin{array}{l}\text { Bile duct } \\
\text { invasion }\end{array}$} & \multirow[b]{2}{*}{ Cholangiectasis } & \\
\hline & Shape & Boundary & & & & & \\
\hline 1 & Oval & Clear & $\mathrm{N}$ & $\mathrm{N}$ & $\mathrm{N}$ & $\mathrm{N}$ & $\mathrm{N}$ \\
\hline 2 & Oval & Clear & $\mathrm{N}$ & $\mathrm{N}$ & $\mathrm{N}$ & $\mathrm{N}$ & $\mathrm{N}$ \\
\hline 3 & Oval & Clear & $\mathrm{N}$ & Right portal vein & $\mathrm{N}$ & $\mathrm{N}$ & $\mathrm{N}$ \\
\hline 4 & Oval & Unclear & $\mathrm{N}$ & $\mathrm{N}$ & $\mathrm{N}$ & $\mathrm{N}$ & $\mathrm{N}$ \\
\hline 5 & Oval & Clear & $\mathrm{N}$ & $\mathrm{N}$ & $\mathrm{N}$ & $\mathrm{N}$ & $\sqrt{ }$ \\
\hline 6 & Oval & Clear & $\mathrm{N}$ & $\mathrm{N}$ & $\mathrm{N}$ & $\mathrm{N}$ & $\mathrm{N}$ \\
\hline 7 & Oval & Clear & $\mathrm{N}$ & Inferior vena cava & $\mathrm{N}$ & $\mathrm{N}$ & $\mathrm{N}$ \\
\hline
\end{tabular}

$\mathrm{N}$, not found; PEComa, perivascular epithelioid cell neoplasm.
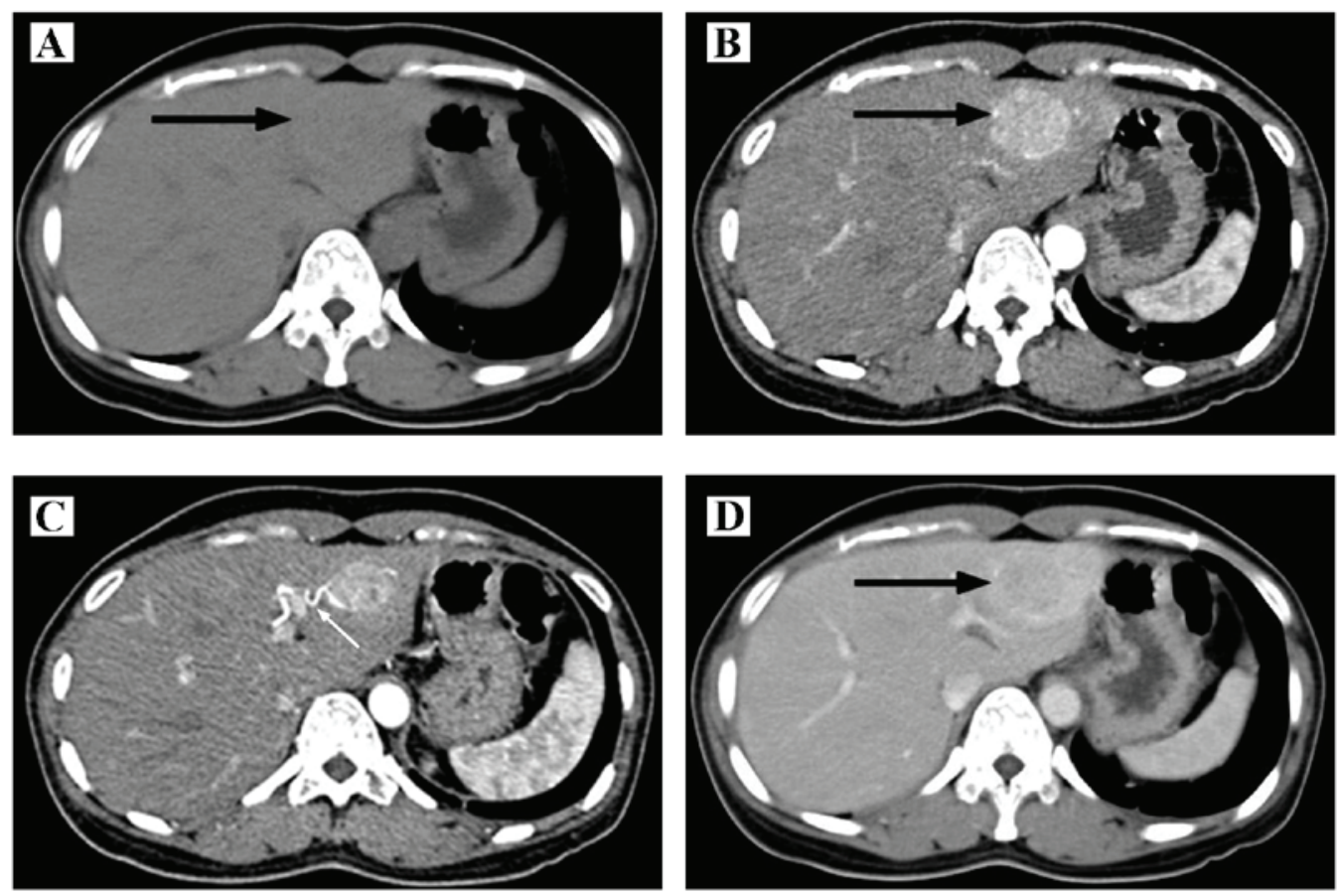

Figure 1. Imaging characteristics of hepatic PEComa. (A) Computed tomography scan, revealing that the lesion is a low-density mass (denoted by the black arrow). (B) Contrast-enhanced CT, revealing a non-homogeneously enhanced lesion during the arterial phase (black arrow). (C) Contrast-enhanced CT shows the curve feeding artery of the lesion during the arterial phase (white arrow). (D) Contrast-enhanced CT shows the lesion enhanced slightly lower than liver parenchyma during portal phase. CT, computed tomography; PEComa, perivascular epithelioid cell neoplasm.
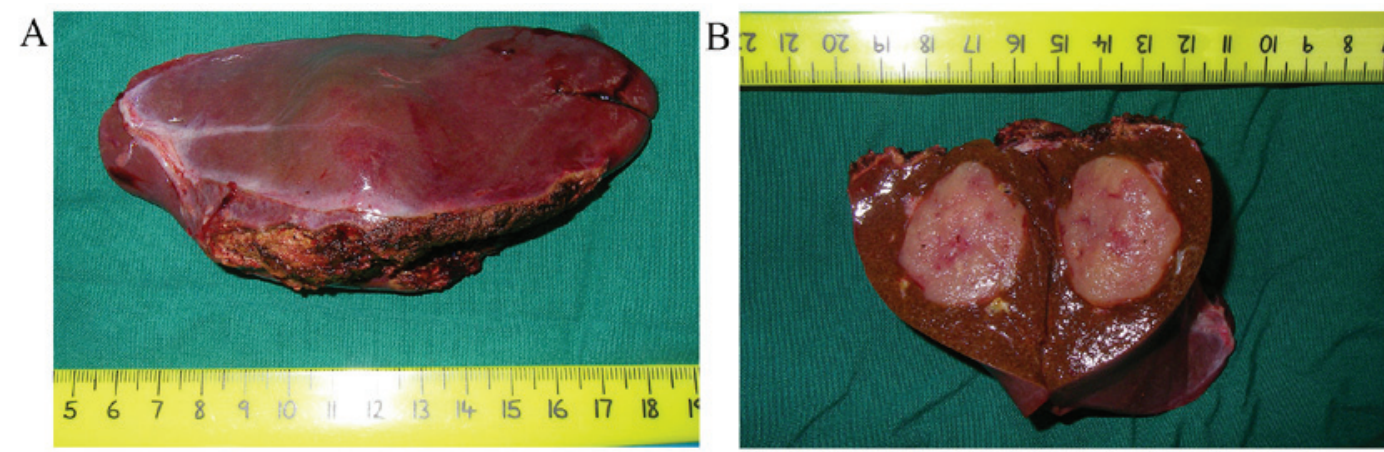

Figure 2. Tumor resection. (A) Resection specimen of Case 5 obtained by partial hepatectomy. (B) The cut surface shows a spherical, well demarcated tumor consisting of yellowish-pink tissue. The lesion is situated within the liver substance, and is not associated with hepatic ligaments. 

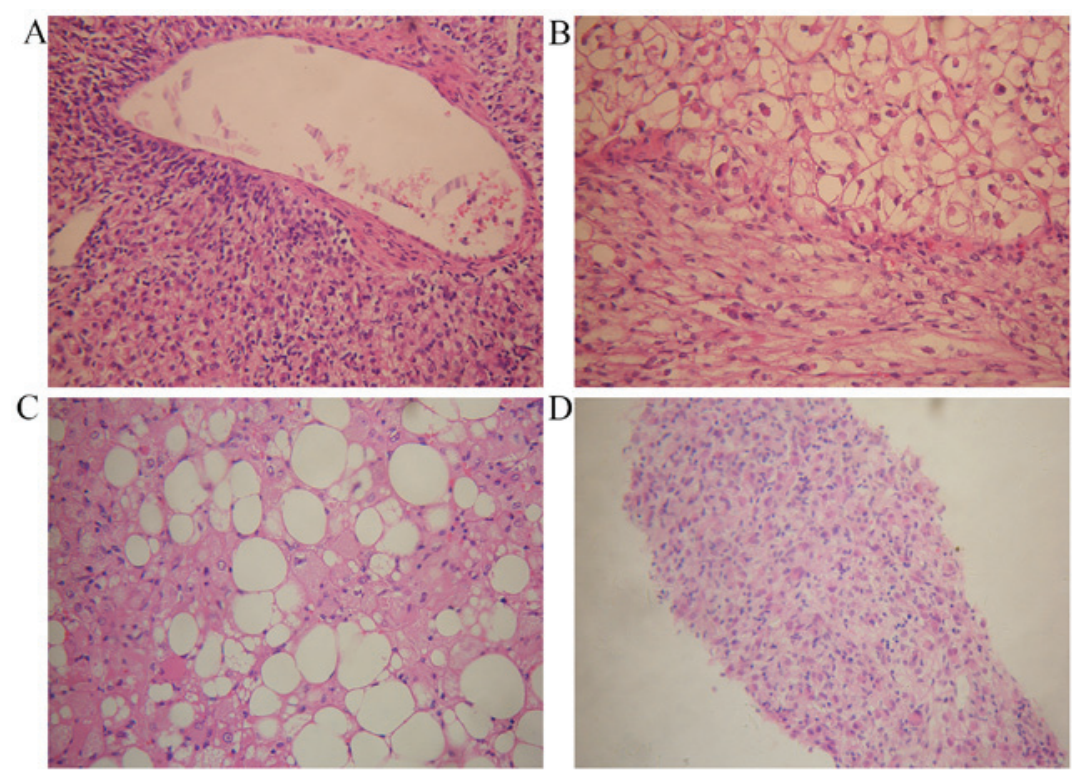

Figure 3. Pathological features of hepatic PEComa. (A) A characteristically thick-walled artery is shown, surrounded by epithelioid cells. (B) The spindled cells and round cells, with a larger size and clear cytoplasm, are shown. (C) Adipocytes intermixed with epithelioid cells. (D) Microscopic findings revealed an abundance of epithelioid cells on FNAB biopsy. All representative images are shown stained with hematoxylin and eosin; magnification, x200.
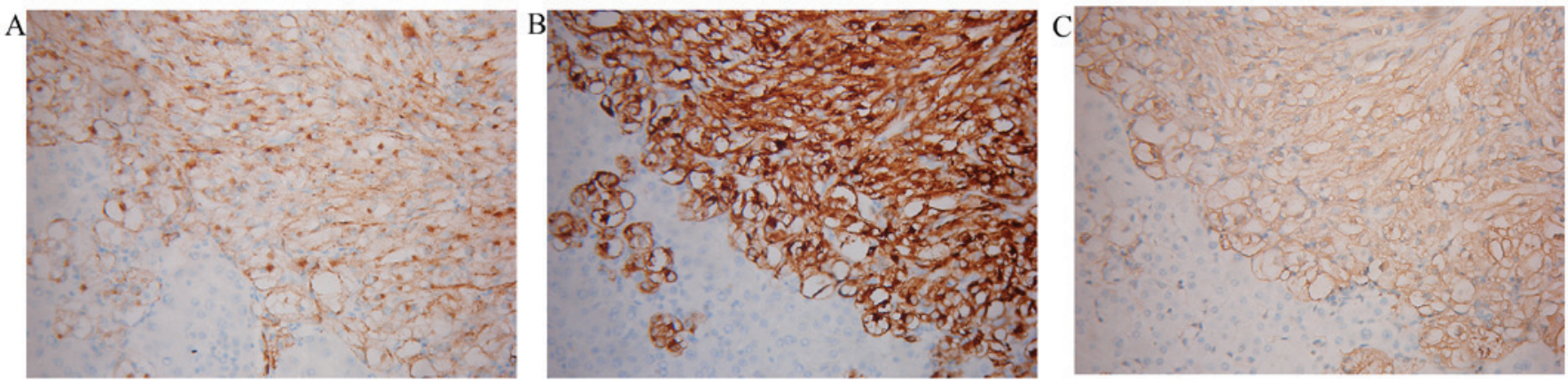

Figure 4. Immunohistochemical analysis. The majority of the tumor cells were markedly immunoreactive for (A) HMB45, (B) Melan-A and (C) smooth muscle $\alpha$-actin. x200.

\section{Discussion}

PEComas are predominantly diagnosed in the kidney, and cases of sporadic hepatic PEComa have been reported worldwide. The majority of cases of hepatic PEComa are found asymptomatically, and have normal serological test results (6), which makes it difficult to diagnose the disease. It has been reported that hepatic PEComas predominantly affect women aged 30-50 years old (5), which is in accordance with our cases. Certain case studies have reported hepatic PEComa presented as acute or chronic abdominal pain $(7,8)$, and 2 patients among our case subjects revealed abdominal pain. However, the majority of them routinely lack specific clinical symptoms and serological abnormalities. Hepatic PEComa may occur as a solitary mass or as multiple lesions, and a higher frequency of multiple masses have been suggested to be associated with tuberous sclerosis (9).

Clinically, a preoperative diagnosis of a hepatic lesion is primarily dependent on imaging examinations. Due to the highly variable histological composition of hepatic PEComa, these tumors often do not possess typical imaging characteristics. On ultrasonography, hepatic PEComa presents as any echogenicity. Early influx into the tumor and rapid drainage of arterial blood to veins, as determined on performing contrast-enhanced ultrasonography, may be a feature of PEComa (10). As mentioned above, adipocytes may be identified intermixing with PECs in varying proportions. Thus, MRI is preferable compared with CT for detecting fat, which appears with a high signal intensity (11). However, it is also difficult to make a diagnosis when the tumors contain a much lower fatty content.

How to make a correct preoperative diagnosis is a topic worthy of investigation. FNAB has been considered to be mandatory for the majority of patients, and histological diagnosis is based on the identification of the different components. Microscopically, epithelioid cells, spindled cells and adipocytes may be identified, which prompt pathologists to take hepatic PEComa into account. Typically, PECs radially arrange around the vascular lumen. They exhibit small, centrally placed, normochromatic, round-to-oval nuclei with small nucleoli, although striking hyperchromasia and nuclear irregularity may be present (1). As noted above, adipocytes 
may also be identified intermixing with PECs in varying proportions. The PECs are characterized by positivity with melanocytic markers and muscle markers (12). The most noteworthy immunological markers include HMB-45, Melan-A and SMA, and negativity for multiple markers, including cytokeratin, CD117, AFP, hepatocytes and chromogranin, confirmed the diagnosis (13).

Due to the rarity of the disease, there are clear difficulties associated with performing a therapeutic trial, and the management of hepatic PEComa remains controversial. The overwhelming majority of reported PEComas reveal a benign pattern. However, there are certain cases that imply invasive growth, with distant metastasis or recurrences (14-16). There is not yet a uniform standard for evaluating the malignant degree of hepatic PEComa. The majority of the reported patients received surgical resection soon after the identification of the tumors, since most of the tumors were preoperatively misdiagnosed as $\mathrm{HCC}$ or hepatic metastasis. Postoperative complications or recurrence are rarely reported. In the present study, 6 patients received surgical treatments, including laparotomic and laparoscopic partial hepatectomy. One patient received TAE and PMCT. None of the patients suffered from postoperative complications or recurrence in the present study. Due to its benign tendency, several researchers have suggested that, when hepatic PEComa is suspected, a fine-needle aspiration combined with HMB-45 staining should be performed in all asymptomatic patients with a lesion $<5 \mathrm{~cm}$ and without serological abnormalities (17). If the diagnosis is confirmed by FNAB and the pathomorphology indicates a benign pattern, careful observation with serial imaging follow-up is recommended $(17,18)$. For a lesion $>5 \mathrm{~cm}$, in cases of progressive enlargement, if the patient has clinical symptoms, or if the FNAB indicates a malignant tendency, a more aggressive approach should be undertaken. Due to the rarity of reports, the exact effects of these therapies have yet to be elucidated. As the majority of previous studies and the present study have shown, patients who undergo surgical resection may expect a good outcome, and therefore surgical resection remains the recommended choice for hepatic PEComa therapy.

\section{Acknowledgements}

This research was supported by a grant from the Natural Science Foundation of China (no. 81572348), the Science and Technology Project of Guangdong Province (no. 2013B021800099) and the Science and Technology Program of Guangzhou (no. 201510010206).

\section{References}

1. Fletcher CDM, Unni KK and Mertens F: Pathology and Genetics of Tumours of Soft Tissue and Bone. Lyon, IARC Press, pp201-202, 2002.

2. Hamilton SR and Aaltonen LA: Pathology and Genetics of Tumours of the Digestive System. Lyon, IARC Press, pp193, 2000.

3. Lu HC, Chau GY and Su CW: Clinical challenges and images in GI. Diagnosis: Hepatic angiomyolipoma mimicking hepatocellular carcinoma. Gastroenterology 136: 1169, 1464, 2009.

4. Xie L, Jessurun J, Manivel JC and Pambuccian SE: Hepatic epithelioid angiomyolipoma with trabecular growth pattern: A mimic of hepatocellular carcinoma on fine needle aspiration cytology. Diagn Cytopathol 40: 639-650, 2012.

5. Zeng JP, Dong JH, Zhang WZ, Wang J and Pang XP: Hepatic angiomyolipoma: A clinical experience in diagnosis and treatment. Dig Dis Sci 55: 3235-3240, 2010.

6. Tryggvason G, Blöndal S, Goldin RD, Albrechtsen J, Bjornsson J and Jonasson JG: Epithelioid angiomyolipoma of the liver: Case report and review of the literature. APMIS 112: 612-616, 2004.

7. Zimmermann A, von der Brelie C, Berger B, Kappeler A and Candinas D: Primary perivascular epithelioid cell tumor of the liver not related to hepatic ligaments: Hepatic PEComa as an emerging entity. Histol Histopathol 23: 1185-1193, 2008.

8. Priola AM, Priola SM, Cataldi A, Marci V and Fava C: Acute abdomen as an unusual presentation of hepatic PEComa. A case report. Tumori 95: 123-128, 2009.

9. Fricke BL, Donnelly LF, Casper KA and Bissler JJ: Frequency and imaging appearance of hepatic angiomyolipomas in pediatric and adult patients with tuberous sclerosis. AJR Am J Roentgenol 182: 1027-1030, 2004.

10. Akitake R, Kimura H, Sekoguchi S, Nakamura H, Seno H, Chiba T and Fujimoto S: Perivascular epithelioid cell tumor (PEComa) of the liver diagnosed by contrast-enhanced ultrasonography. Intern Med 48: 2083-2086, 2009.

11. Prasad SR, Wang H, Rosas H, Menias CO, Narra VR, Middleton WD and Heiken JP: Fat-containing lesions of the liver: Radiologic-pathologic correlation. Radiographics 25: 321-331, 2005.

12. Folpe AL and Kwiatkowski DJ: Perivascular epithelioid cell neoplasms: Pathology and pathogenesis. Hum Pathol 41: 1-15, 2010.

13. Martignoni G, Pea M, Reghellin D, Zamboni G and Bonetti F: PEComas: The past, the present and the future. Virchows Arch 452: 119-132, 2008.

14. Dalle I, Sciot R, de Vos R, Aerts R, van Damme B, Desmet V and Roskams T: Malignant angiomyolipoma of the liver: A hitherto unreported variant. Histopathology 36: 443-450, 2000.

15. Nguyen TT, Gorman B, Shields D and Goodman Z: Malignant hepatic angiomyolipoma: Report of a case and review of literature. Am J Surg Pathol 32: 793-798, 2008.

16. Kamimura K, Oosaki A, Sugahara S, Mori S, Moroda T, Satoh O, Morita T, Kimura K, Kamura T, Nomoto M and Aoyagi Y: Malignant potential of hepatic angiomyolipoma: Case report and literature review. Clin J Gastroenterol 3: 104-110, 2010.

17. Yang L, Xu Z, Dong R, Fan J, Du Y, Zhang Y, Wang X, Cheng X and Guo J: Is surgery necessary for patients with hepatic angiomyolipoma? Retrospective analysis from eight Chinese cases. J Gastroenterol Hepatol 28: 1648-1653, 2013.

18. Belghiti J, Cauchy F, Paradis V and Vilgrain V: Diagnosis and management of solid benign liver lesions. Nat Rev Gastroenterol Hepatol 11: 737-749, 2014. 